\title{
AmAZGNÁNAS
}

Revista científica del Amazonas

Volumen 3 Número 5

Enero-junio 2020

\section{Perfeccionamiento de la estrategia curricular de medio ambiente de la carrera de ciencias farmacéuticas de la Universidad de Oriente, Cuba.}

\author{
Improvement of the Environmental Curricular Strategy of the Pharmaceutical \\ Sciences Career at the Universidad de Oriente, Cuba. \\ Melhoria da Estratégia Curricular Ambiental da Carreira em Ciências \\ Farmacêuticas na Universidade de Oriente, Cuba.
}

Recibido: 15 de octubre de 2019
Aceptado: 10 de diciembre de 2019

Escrito por:

Dra. C. Idelsy Chil Núñez ${ }^{1,2}$

https://orcid.org/0000-0003-4661-0472

Dr. C. Julio César Escalona Arranz ${ }^{1}$

https://orcid.org/0000-0003-3609-4451

MSc. Silvia del Carmen Molina Bertrán ${ }^{1}$ https://orcid.org/0000-0003-1560-0191

Dr. C. Carlos Manuel Dutok Sánchez ${ }^{3}$ https://orcid.org/0000-0001-6712-3185

MSc. Daily Arias Ramos ${ }^{1}$ https://orcid.org/0000-0003-0128-9558

MSc. Liana Pérez Rondón ${ }^{1}$ https://orcid.org/0000-0001-8063-5476

Dra. C. Ania Ochoa Pacheco ${ }^{1}$ https://orcid.org/0000-0002-1028-6626

Dr. C. Raimundo Nonato Picanço Souto ${ }^{2}$ https://orcid.org/0000-0002-8795-1217

\section{Resumen}

La Educación ambiental se enfoca en los aspectos relacionados a cómo continuar el desarrollo al mismo tiempo que se protege, preserva y conserva los sistemas de soporte vital del planeta. Para poder garantizar el enfoque sistémico y multidisciplinario de los aspectos relacionados con este tipo de educación es imprescindible realizar en los colectivos, actividades metodológicas para establecer un sistema para la enseñanza de sus diferentes métodos, instrumentos y metodología que permitan lograr una mayor preparación de los estudiantes en la solución a los problemas de su esfera profesional. Es por ello que el objetivo del presente trabajo es perfeccionar la Estrategia de Medio Ambiente de la Carrera de Ciencias Farmacéuticas desde el enfoque Ciencia-TecnologíaSociedad en aspectos relacionados con investigaciones realizadas sobre el uso de insecticidas de menor riesgo para la salud humana y el medio ambiente. Como punto de

\footnotetext{
${ }^{1}$ Departamento de Farmacia. Facultad de Ciencias Naturales y Exactas. Universidad de Oriente. Santiago de Cuba. Cuba.

${ }^{2}$ Departamento de Biologia e Ciências da Saúde. Laboratório de Arthropoda. Universidade Federal do Amapá. Macapá. Brasil.

${ }^{3}$ Núcleo de Ciências Fisiológicas, Universidade Federal do Amapá, Campus Binacional do Oiapoque, Amapá, Brasil.
} 
partida, se tomó el desarrollo de insecticidas botánicos a partir de plantas medicinales, que es una de las líneas de investigación del Departamento de Farmacia de la Universidad de Oriente, que evalúa la eficacia de diferentes plantas del Oriente de Cuba para el control alternativo de moscas y mosquitos de importancia médico-sanitaria, demostrando la efectividad y no toxicidad de estos extractos vegetales, representando estas sustancias un método alternativo eficaz. Se revisó la Estrategia Curricular de Medio Ambiente de la Carrera y se incluyeron en estas actividades relacionadas con las investigaciones realizadas sobre el uso de insecticidas ecológicos en seis asignaturas pertenecientes tanto al currículo base como optativo.

Palabras claves: Educación ambiental, estrategia curricular, extractos vegetales, insecticidas, medio ambiente.

\begin{abstract}
Environmental education is education on how to continue development while protecting, preserving and conserving the life support systems on the planet. In order to guarantee a systemic and multidisciplinary approach on environmental education, it is essential to perform methodological activities establishing a system of multiple methods, instruments and methodologies on the teaching groups, allowing the achievement of higher preparation levels on the students in the solution to their professional needs. That is why the objective of this work is to improve the Environmental Strategy of the Pharmaceutical Sciences Career at Universidad de Oriente, from a science-technologysociety approach, in aspects related to researches carried on the use of bio-insecticides as alternative for diminish their risk on human health and environment. The development of botanical insecticides from medicinal plants was taken as a starting point as one of the research lines from the before mentioned Pharmacy Department. In this topic, several plants growing up in the Eastern of Cuba are evaluated in their effectiveness as alternative control of flies and mosquitoes with medical-sanitary relevance, demonstrating at the same time it effectiveness and non-toxicity, and in consequence; allowing it use as an effective alternative method. The Career Environmental Education Strategy was reviewed, and activities related to this research topic were included in six subjects belonging to the base and optional curriculums.
\end{abstract}

Keywords: environmental education, curricular strategy, plant extracts, insecticides, environment.

\title{
Resumo
}

Educação ambiental é a áerea de ensino que visa o desenvolvimento, porém de forma sustentável, protegendo, conservando e preservando os sistemas de suporte à vida do planeta. Para garantir a abordagem sistêmica e multidisciplinar dos aspectos relacionados à educação ambiental, é essencial a realização de atividades metodológicas em grupos para estabelecer um sistema de ensino dos diferentes métodos, instrumentos e metodologias que permitam preparar os problemas dentro de suas repectivas atividades profissionais. Por isso, o objetivo do presente trabalho foi contribuir e aperfeiçoar a Estratégia Ambiental do Curso de graduação em Ciências Farmacêuticas a partir da abordagem ciência- tecnologia-sociedade nos aspectos relacionados às pesquisas 


\section{AMAZÓNAS}

realizadas com o uso de inseticidas de menor risco à saúde humana e danos ao meio ambiente. $\mathrm{O}$ desenvolvimento de inseticidas botânicos a partir de plantas medicinais é tomado como ponto de partida, é sendo uma linha de pesquisa do Departamento de Farmácia da Universidade de Oriente que avalia a eficácia de diferentes plantas no leste de Cuba, no controle alternativo de moscas e mosquitos de importância médico-sanitária, demonstrando a eficácia e a não-toxicidade desses óleos e extratos vegetais, para que essas substâncias possam representar um método alternativo eficaz. A Estratégia de Educação Ambiental do Curso de graduação foi revisada e foram incluídas atividades relacionadas as pesquisas realizadas sobre o uso de inseticidas ecológicos em seis disciplinas pertencentes ao currículo básico e optativo.

Palavras-chave: educação ambiental, estratégia curricular, extratos vegetais, inseticidas, meio ambiente.

\section{Introducción}

La educación ambiental es educación sobre cómo continuar el desarrollo al mismo tiempo que se protege, preserva y conserva los sistemas de soporte vital del planeta; de cómo las acciones individuales y de grupo pueden influenciar la relación entre calidad de vida humana y la condición del ambiente (Marin, 2007). Es un proceso educativo orientado a desarrollar actividades, valores y conocimientos en los estudiantes, logrando la participación de los mismos en la protección del medio ambiente; además de constituir un proceso de cambio, de percepción del hombre hacia su entorno y de una voluntad de entender que a partir de este hay que actuar. En tal sentido, la este tipo de educación, se convierte en un factor necesario en la formación cultural y ambiental del futuro egresado de la carrera de Ciencias Farmacéuticas, la cual tendrá su materialización en las diferentes formas de trabajo científico y docente-educativo propias de las formas de enseñanza de la carrera.

En el proceso de formación de los profesionales, la cultura ambiental es parte de la educación integral que se desarrolla en los futuros egresados, de modo que su actuación científica y tecnológica se revierta en el logro de una mejor calidad de vida a la población con un uso óptimo de los recursos naturales, humanos y financieros disponibles. La estrategia curricular de medio ambiente constituye la guía de acción que en cada carrera que se cursa en la Universidad de Oriente en Santiago de Cuba, se elabora y aplica para lograr tales aspiraciones (ESTRATEGIA CURRICULAR DE MEDIO AMBIENTE, 2018).

Uno de los problemas ambientales de gran impacto en la actualidad es el cambio climático, que se adjudica directa o indirectamente a las actividades humanas en el planeta, pero sus efectos son regionales y locales. La causa principal es la aplicación de patrones de desarrollo basados en el consumo desenfrenado de combustibles fósiles y recursos naturales que decrecen en el tiempo y traen como consecuencia el aumento de las concentraciones de gases de efecto invernadero en la atmósfera, lo que provoca un incremento de la temperatura del planeta, entre otros efectos (Ibárcena yScheelje, 2003; Gerard, 2009; Useros, 2013). Otro factor importante que afecta el medio ambiente es el uso de sustancias tóxicas, como los insecticidas químicos (sintéticos) para el control de insectos, los cuales afectan a los humanos y a animales, contaminando el aire, el agua y 


\section{AMAZONAS}

la cadena alimentaria (Mendonça et al., 2011). El uso excesivo de estos tipos de insecticidas también ha resultado en una resistencia progresiva de las plagas a estos químicos, reduciendo su efectividad y generando consecuencias con potenciales negativos como una mayor frecuencia de uso, dosis y mezclas con compuestos más tóxicos (Hemingway y Ranson, 2000).

Para poder garantizar el enfoque sistémico y multidisciplinario de los aspectos relacionados con la Educación Ambiental, es imprescindible realizar en los colectivos, actividades metodológicas para establecer un sistema para la enseñanza de sus diferentes métodos, instrumentos y metodología, que permita lograr una mayor preparación de los estudiantes y como futuros egresados sean capaces de utilizar estos conocimientos en la solución a los problemas de su esfera profesional.

\section{Objetivo general}

Perfeccionar la Estrategia Curricular de Medio Ambiente de la carrera de Ciencias Farmacéuticas desde el enfoque ciencia-tecnología-sociedad aplicando las investigaciones realizadas sobre el uso de insecticidas de menor riesgo para la salud humana y el medio ambiente.

\section{Marco teórico}

La educación ambiental es un proceso que dura toda la vida y que tiene como objetivo impartir conciencia ambiental, conocimiento ecológico, actitudes y valores hacia el medio ambiente para tomar un compromiso de acciones y responsabilidades que tengan por fin el uso racional de estos recursos y poder lograr así un desarrollo adecuado y sostenible.

Los objetivos de este tipo de educación vienen definidos por la UNESCO y son los siguientes:

Toma de conciencia: concienciar a las personas de los problemas relacionados con el medio ambiente.

- Conocimientos: ayudar a interesarse por el medio ambiente.

- Actitudes: adquirir interés por el medio ambiente y voluntad para conservarlo.

- Aptitudes: ayudar a adquirir aptitudes para resolver el problema.

- Capacidad de evaluación: evaluar los programas de Educación Ambiental.

- Participación: desarrollar el sentido de la responsabilidad para adoptar las medidas adecuadas (Marin, 2007).

La educación ambiental es una apuesta de las naciones, con el objetivo de contribuir al desarrollo sostenible y la preservación de los recursos naturales. En este sentido, las Naciones Unidas declaró la década de la educación para un futuro sostenible (2005-2014), con el propósito de "contribuir a la formación de ciudadanas y ciudadanos conscientes de los problemas socio-ambientales a los que se enfrentan y preparados para participar en la toma de decisiones fundamentadas" (Línea Verde: La Educación Ambiental). 


\section{AMAZÚNAS}

Esta educación en el aula debe de ser transdisciplinaria, desde la perspectiva científica (ecología, biología), ideologista (valores, comportamientos, actitudes) e integradoras (aprendizaje, educación). El cambio exige la transición de una concepción simple a una más compleja del medio, supone reestructurar las propias creencias del pensamiento cotidiano. Con propuestas de carácter integrador y globalizador, donde la educación ambiental desarrolla una visión del mundo más ambiental (Nieto, 2004).

Los actuales hábitos de consumo, las formas de producción industrial, los métodos de explotación de recursos, el aumento de los desechos, entre otros, ejercen una presión tan grande sobre los ecosistemas que superan su resiliencia o capacidad de recuperación. Cuando esta situación se prolonga, genera una crisis ambiental, repercutiendo en el sistema productivo arrastrando consigo la crisis energética y siendo la energía el corazón del crecimiento económico, su escasez está relacionada directamente con crisis económicas de gran magnitud (Vilches et al., 2009).

El cambio climático, con la consecuente agudización de los períodos de sequía, la ocurrencia de lluvias intensas, la elevación de la temperatura y el incremento de las penetraciones del mar, así como de la intensidad y frecuencia de los fenómenos extremos como los huracanes, se reconoce como una de las causas importantes de la pérdida de la diversidad biológica en Cuba (Planos et al., 2012). Siendo una realidad que las sequías se intensifican, así como que la temperatura aumenta y el mar se eleva, por lo que en vista a los impactos negativos que puede tener el cambio climático (Iturralde-Vinent y Serrano, 2015), se han tomado medidas para mitigar estos efectos en el archipiélago, creándose diferentes alternativas, entre la que se destaca la creación del Plan de Acciones para la Adaptación y Mitigación del Cambio Climático en el año 2017, más conocido como "Tarea Vida" por el Ministerio de Ciencia, Tecnología y Medio Ambiente (CITMA) de Cuba.

En el tema del medio ambiente, la ciencia y la tecnología han jugado un doble papel en la forma de abordarlo. En primer lugar, se ha culpado a la ciencia y a la tecnología de provocar la mayor parte de los problemas que actualmente aquejan al planeta. En segundo lugar, se le otorga la responsabilidad de crear soluciones para estos problemas, a partir de la creación de nuevas tecnologías, del cambio en los patrones de consumo y de la exploración de alternativas para la satisfacción de las necesidades de las personas. Ante la ocurrencia de desastres relacionados con el desarrollo industrial, como los derrames de petróleo o catástrofes nucleares, se comienza a formar una concienciación colectiva acerca de los riesgos e impactos que podrían generar una ciencia y tecnología fuera de control. Igualmente, los fenómenos como el cambio climático, la contaminación de las fuentes de agua y la pérdida de biodiversidad, entre otros, han llamado la atención sobre la necesidad de desarrollar tecnologías que produzcan mínimos daños al medio ambiente. A la vez que se privilegie el uso de estas tecnologías frente aquellas que generan un alto impacto negativo al medio ambiente (Avellaneda, 2002).

Envueltos en esta problemática se encuentran los insecticidas químicos de origen sintético, que surgieron a mediados de la década de 1940. Estos productos actúan en el sistema nervioso de los insectos causando su muerte (Parra, 2010). Es el medio de control más utilizado para dípteros sinantrópicos, constituido principalmente por organoclorados y organofosforados (Buse y Park-Brown, 2018). El uso de los insecticidas químicos 
(sintéticos) para el control de insectos es peligroso. Se ha reconocido que el uso excesivo de estos tipos de compuestos resulta en una progresiva resistencia de las plagas a estos químicos, disminuyendo su efectividad y generando consecuencias con potenciales negativos, como el aumento de la frecuencia de uso, de la dosis y de mezclas con compuestos más tóxicos, además han perdido su efectividad a medida que las poblaciones objetivo adquieren resistencia genética contra sus compuestos (WHO, 1990; Environews Forum, 1999; Aktar, Sengupta y Chowdhury, 2009).

La resistencia a insecticidas químicos se extiende por todo el mundo y afecta a la mayoría de las clases químicas disponibles. Es cierto que la resistencia a los insecticidas sintéticos más modernos (neonicotinoides, fenilpiraciones, spinosad, etc.) es relativamente poco frecuente, pues estos compuestos no han sido muy utilizados ${ }^{9}$. Las poblaciones multirresistentes aumentan cada vez más. Estas son resistentes simultáneamente a más de una clase química (por ejemplo, organofosforados, carbamatos y piretroides al mismo tiempo(Georghiou, 1994; David, 2007).

Todo lo expuesto hasta ahora incrementa el interés por los insecticidas alternativos por lo que las investigaciones que permiten desarrollar y usar productos botánicos para el manejo de plagas están nuevamente aumentando en los últimos años (Vieira, Mafezoli y Biavatti, 2001). Este interés viene al encuentro de la necesidad de buscar métodos alternativos de menor impacto o riesgos para la salud humana y el medio ambiente, así como por la creciente demanda de productos alimenticios saludables y exentos de residuos de agrotóxicos.

Son innumerables las plantas poseedoras de poderes insecticidas, que deberían no sólo ser investigadas, sino también ser utilizadas como modelos para la síntesis de nuevos principios activos. Los conocimientos adquiridos con los mecanismos de defensa de las plantas han ayudado en el desarrollo de los métodos de control de plagas menos agresivas al ambiente. Muchos de los principios activos de las plantas tienen acción específica para algunos grupos de organismos, sin afectar a otros y esta característica es importante para controlar sólo los organismos blancos (Menezes, 2005). Esto demuestra la importancia del desarrollo de los insecticidas botánicos a partir de las plantas medicinales, resultando esta una línea de investigación del Departamento de Farmacia de la Universidad de Oriente que evalúa la eficacia de diferentes plantas del Oriente de Cuba, como Ocimum sanctum var. cubensis, Pouteria mammosa (L.) Cronquist y Persea americana Mill; para el control alternativo de moscas y mosquitos de importancia médico-sanitaria.

Es necesario que la sociedad reciba una educación integral que permita promover una conciencia crítica a través de un enfoque interdisciplinario entre ciencia, tecnología y sociedad, donde se puedan descubrir las posibilidades para minimizar el impacto ambiental de nuestras acciones y poder desarrollar habilidades para transformar el entorno haciendo uso de tradiciones, valores y hábitos, que los hombres han desarrollado a lo largo de la historia. Desde el ámbito universitario se debe realizar trabajo en este sentido desde lo curricular, no solo en los contenidos impartidos, sino también desde las Estrategias Curriculares, fundamentalmente la de MEDIO AMBIENTE. 


\section{AMAZÓNAS}

\section{Métodos}

Se realizó un análisis del contenido de la Estrategia Curricular de Medio Ambiente, basado en la lectura textual de ese documento como instrumento de recogida de información. Esta lectura se realizó siguiendo el método científico, es decir, de manera sistemática, objetiva, replicable y válida (Andréu, 2018).

Se analizaron también diversos informes científicos elaborados por el Grupo de Investigación de Productos naturales del departamento de Farmacia de la Universidad de Oriente, donde se comprobó la existencia de ensayos referidos al tamizaje fitoquímico y técnicas de análisis cromatográfico para realizar la caracterización química cualitativa y cuantitativa de los extractos y aceites esenciales obtenidos de las plantas. También se llevó a cabo la evaluación de estos a diferentes concentraciones) hasta en 6 especies de Dípteros muscoides de las familias Caliphoridae (Cochliomyia macellaria, Chrysomya putoira, Chrsomya albiceps, Chrysomya megacephala, Lucilia cuprina) y Muscidae (Musca domestica), teniendo en cuenta los parámetros: influencia de estos sobre el desarrollo pos-embrionario al ser aplicados de forma tópica sobre las larvas; influencia de los extractos en el peso de las larvas y sobre el sexo de la descendencia; influencia sobre la mortalidad de los insectos y el efecto adulticida. Además, se evaluó la posible toxicidad de estos compuestos mediante los estudios de Toxicidad Aguda Oral, Toxicidad Dérmica Aguda e Irritabilidad Oftálmica. Se evaluaron también los resultados de algunas de estas sustancias frente a mosquitos adultos de Aedes aegypti Linnaeus, Culex quinquefasciatus Say y Anopheles albitarsis s.l.

Los resultados mostraron la marcada influencia de todos los extractos evaluados sobre el desarrollo pos-embrionario de las moscas, así como la influencia sobre el peso y la razón sexual. Se demostró el efecto adulticida en moscas y mosquitos con mejores resultados para el aceite esencial. Finalmente se comprobó la no toxicidad de los extractos vegetales y el aceite esencial utilizados en la investigación, por lo que estas sustancias pueden representar un método alternativo eficaz para controlar estas especies de moscas y mosquitos, constituyendo insecticidas botánicos de menor riesgo para la salud humana y el medio ambiente (Carriço et al., 2014; Dutok et al., 2015; Chil et al., 2017; 2018; Molina et al., 2018; Chil et al., 2019; Souto et al. en prensa).

Teniendo en cuenta que la educación ambiental requiere de estrategias que permitan al educador canalizar acciones que conduzcan a nuevas formas de relaciones (comportamiento, conducta) sustentables de la población con el ambiente natural. Estas deben estar encaminadas a la participación de las personas de manera que puedan comprender y transformar su realidad, para actuar solidariamente en la solución de los problemas. Por consiguiente, la educación ambiental juega un papel muy importante en la generación de estrategias para solucionar los problemas socio-ambientales que sufren las comunidades (Martínez, 2012). Se recomienda incluir en la Estrategia Curricular de Medio Ambiente de la carrera de Licenciatura en Ciencias Farmacéuticas las siguientes acciones. 


\section{AMAZƠNAS}

\section{Acciones a realizar}

Estas investigaciones se incorporarán a la Estrategia Curricular de Medio Ambiente de la carrera de Ciencias Farmacéuticas, para que, desde las diferentes asignaturas del Plan de Estudio, se tome como punto de partida para el desarrollo de otras tareas que permitan analizar y brindar soluciones a la problemática de los insecticidas químicos desde la óptica de los productos naturales, con énfasis en las plantas medicinales. Esta tiene como OBJETIVO:

Formar un profesional farmacéutico, con una cultura ambiental que les permita utilizar estos conocimientos en la solución de problemas concretos de sus diferentes esferas de actuación profesional.

Se realizarán las siguientes tareas:

\section{SEGUNDO AÑO}

\section{Asignatura: Farmacognosia y Química de los Productos Naturales}

\section{Actividades:}

1. Reconocer las plantas medicinales que contengan metabolitos activos, que puedan emplearse como insecticidas botánicos, para de esta forma contribuir al desarrollo y uso racional de la Medicina Natural y Tradicional en el Sistema de Salud y su protección al ecosistema como parte del Programa de Protección al Medio Ambiente en nuestro país.

Formas de control: Se chequeará en la discusión de artículos en seminarios, conferencias y en la defensa del trabajo de curso de esta asignatura.

Responsables: Profesores de la asignatura.

Asignatura: Problemas Sociales de la Ciencia y la Tecnología

\section{Actividades:}

1. Debates en Conferencias y seminarios sobre el desarrollo de una cultura medio ambiental, haciendo énfasis en el peligro del uso de insecticidas sintéticos.

Formas de control: Conferencias, seminarios y estudios independientes.

Responsables: Profesores de la asignatura

\section{CUARTO AÑO}

Asignatura: Química Medicinal I y II 


\section{AMAZÓNAS}

\section{Actividades:}

1. Discutir en los tipos de clases que lo requieran como determinadas sustancias influyen en la contaminación ambiental, causan daños al ecosistema y buscar posibles soluciones.

2. Analizar en conferencia y seminarios los aspectos de relación estructura químicaactividad biológica de determinados compuestos químicos de origen natural, que posean actividad insecticida.

Responsables: Profesores de la asignatura.

Formas de control: Seminarios, conferencias y laboratorios.

$\underline{\text { Asignatura: Terapia con productos naturales (Optativa) }}$

\section{Actividades:}

1. Documentar al estudiante con la forma de utilización terapéutica correcta de las plantas medicinales, incluyendo su uso como insecticida, garantizando el uso racional de las mismas, permitiendo la conservación de la flora autóctona y evitando las posibles consecuencias sobre el medio ambiente.

Formas de control: Discusión del trabajo final de la asignatura. Actividades prácticas.

Responsable: Profesores de la asignatura.

\section{$\underline{\text { Asignatura: Toxicología }}$}

\section{Actividades:}

1. Vincular los contenidos recibidos a los diferentes problemas de contaminación ambiental, haciendo énfasis en los insecticidas sintéticos y la relación del hombre con su entorno.

2. Resolver los ejercicios de seminarios relacionados con los mecanismos moleculares por los cuales determinados contaminantes ambientales causan daño al hombre y sus posibles tratamientos.

Forma de control: a través de seminarios y clases prácticas.

Responsable: Profesor de la asignatura.

\section{Conclusiones}

Se incluyeron actividades en la Estrategia de Medio Ambiente de la carrera de Ciencias Farmacéuticas desde el enfoque Ciencia-Tecnología-Sociedad en aspectos relacionados con las investigaciones realizadas sobre el uso de insecticidas de menor riesgos para la salud humana y el medio ambiente en seis asignaturas pertenecientes tanto al currículo base como optativo. 


\section{Declaración de conflicto de interés}

Los autores declaran que no tienen ningún conflicto de interés.

\section{Referencias bibliográficas}

AktarMd W, Sengupta D, Chowdhury A. (2009). Impact of pesticides use in agriculture: their benefits and hazards. InterdiscToxicol.; 2(1): 1-12.

Andréu Abela, J. (2018). Las técnicas de Análisis de Contenido: Una revisión actualizada. Disponible en: http://mastor.cl/blog/wp-content/uploads/2018/02/Andreu.-analisisde-contenido.-34-pags-pdf.pdf

Avellaneda Cusaría, A. (2002). Gestión ambiental y planificación del desarrollo. El Reloj verde. Entropía-globalización-democracia-. cultura, Bogotá: Ecoe Ediciones.

Buse, A., Park-Brown, S. G. (2018). Natural products for insect pest management. Gainesville: UF/IFAS, 2002. Disponível em: http://edis.ifas.ufl.edu/IN197 Consultado en: 15 enero 2019.

Carriço, C. Pinto Z T., Dutok C. M.S., Caetano R L., Pessanha R., Chil-Nuñez I.,. Mendonça PM, Escalona-Arranz J C., Reyes-Tur, B. Queiroz M. M.C. (2014). Biological activity of Pouteriasapota leaf extract on postembryonic development of blowfly Chrysomyaputoria (Wiedemann, 1818) (Calliphoridae). RevBras Farma; 24 304-308.

Chil Núñez I, Escalona Arranz JC, Berenguer Rivas CA, Mendonça PM, Mateo Pérez K, Dutok Sánchez CM, Cortinhas LB, Silva CF, Carvalho MG, Queiroz MMC. (2017). Chemical Composition and Toxicity of Ocimum sanctum L. var. Cubensis Essential Oil Up-Growing in the Eastern of Cuba. International Journal of Pharmacognosy and Phytochemical Research 9(7): 1021-1028.

Chil Núñez I, Mendonça PM, EscalonaArranz JC, Cortinhas LB, Dutok Sánchez CM, Queiroz MMC. (2018). Insecticidal effects of Ocimum sanctum var. cubensis essential oil on the diseases vector Chrysomyaputoria. Journal of Pharmacy \&Pharmacognosy Research 6 (3): 148-157.

Chil-Núñez I, Mendonça PM, Escalona-Arranz J C., Dutok-Sánchez C M., Souto R.N.P, Queiroz M.M.C. (2019). Actividad insecticida del aceite esencial de Ocimumsanctumvar. cubensis en el control de Cochliomyiamacellaria (Fabricius), en condiciones de laboratorio. Revista científica del Amazonas, 2 (4), Julio - diciembre 2019, p41-56.

David G, Linda J, GahancSimon W, BaxteraJian-ZhouZhao, Anthony M, SheltonFre, Goulde Bruce, Tabashnikd E. (2007). The diversity of Bt resistance genes in species of Lepidoptera. Journal of Invertebrate Pathology.; 95 (3) July: 192-197.

Dutok C.M. S., Berenguer-Rivas CA, Rodríguez-Leblanch E., Pérez-Jackson L., ChilNuñez I., Escalona-Arranz J C, Reyes-Tur B. y Queiroz M. M. C. (2015). Acute Toxicity and Dermal and Eye Irritation ofthe Aqueous and Hydroalcoholic Extracts of the Seeds of (Zapote) Pouteriamammosa (L.) Cronquist. The Scientific World Journal. Article ID 642906, 7 pages http://dx.doi.org/10.1155/2015/642906.

Environews Forum. Killer environment. Environ Health Perspect. 1999; 107 (2): 62-63. ESTRATEGIA CURRICULAR DE MEDIO AMBIENTE. (2018). Universidad de Oriente. Facultad de Ciencias Naturales y Exactas. Departamento de Farmacia. Santiago de Cuba, Cuba. 


\section{AMAZÚNAS}

Georghiou GP. (1994). Principles of insecticideresistancemanagement. Phytoprotection; 75 (4): 51-59.

Gerard Jori. (2009). EL CAMBIO CLIMÁTICO COMO PROBLEMA Y EL DIÁLOGO SOCIAL COMO SOLUCIÓN.Investigaciones geográficas, nº 48, pp. 125 - 160.ISSN: 0213-4691.

Hemingway J, Ranson H. (2000). Insecticideresistance in insectvectors of human disease. Annu. Rev. Entomol. 45:371-391.

Ibárcena Escudero M., Scheelje Bravo, J M. (2003). EL CAMBIO CLIMÁTICO PRINCIPALES CAUSANTES, CONSECUENCIAS Y COMPROMISOS DE LOS PAÍSES INVOLUCRADOS. http://www.fao.org/3/XII/0523-B2.htm

Iturralde-Vinent y Serrano. (2015). Peligros y vulnerabilidades de la zona marino-costera de Cuba: estado actual y perspectivas ante el cambio climático hasta el 2100, ISBN 9592703388, 9789592703384.

Linea Verde: La Educación Ambiental. Disponible en: http://www.lineaverdemunicipal.com/consejos-ambientales/educacion-am

Marin C. 2007. Educación ambiental. Disponibe en: http://laeduambiental.blogspot.com/ Martínez Castillo R. (2012). ENSAYO CRITICO SOBRE EDUCACIÓN AMBIENTAL. Revista Electrónica Diálogos Educativos, 24(12), 74-104.

Mendonça PM, Lima MG, Albuquerque LRM, Carvalho MG e Queiroz MMC. (2011). Effects of latex from "Amapazeiro" Parahacorniaamapa (Apocynaceae) on blowfly Chrysomyamegacephala (Diptera: Calliphoridae) post-embryonic development. VeterinaryParasitology. 178:379-382.

Menezes ELA. (2005). Inseticidas Botânicos: Seus Princípios Ativos, Modo de Ação e Uso Agrícola. Empresa Brasileira de Pesquisa Agropecuária. Centro Nacional de Pesquisa em Agrobiologia. Ministério da Agricultura, Pecuária e Abastecimento. Seropédica - RJ.

Molina, S.C., Mendonça, P.M., Reyes, B., Queiroz, M.M.C., Escalona, J.C., García, J., Guisado, F. (2018). Effects of Persea americana Mill. seed extracts on the postembryonic development of Muscadomestica (Diptera: Muscoide). Journal of Pharmacy\&PharmacognosyResearch, 6 (2), 96-107.

Nieto-Carabeo, L.M. (2004). ¿Cuál es el papel de la Educación Ambiental? Revista Universitarios. Editorial Universitaria Potosí (Mex.) 12(2): 56-61. Disponible en: http://ambiental.uaslp.mx/

Parra A, Cadena Z. (2010). The environment and the relations of science, technology and society: a general overview. CS No. 6, 331 - 359, julio-diciembre. ISSN 2011-0324.

Planos Gutiérrez et al. Impacto del cambio climático y medidas de adaptación en Cuba, 2012, Eds. La Habana, Cuba.

Souto RNP, Escalona-Arranz JC, Chil-Núñez I, García-Diaz J, Ferreira RMA, da Costa T. S. De la Vega J, Heredia-Diaz Y, Fernández-González R, Queiroz MMC. Chemical composition and adulticidal activity of Cuban plants essential oils against three mosquitoes' vector species. (Artículo en arbitraje).

Tarea Vida. Ministerio de Ciencia Tecnología y Medio Ambiente (CITMA) https://www.ecured.cu/Tarea_Vida

Useros Fernández, J L. (2013). EL CAMBIO CLIMÁTICO: SUS CAUSAS Y EFECTOS MEDIOAMBIENTALES CLIMATE CHANGE: CAUSES AND ENVIRONMENTAL EFFECTS. An Real AcadMedCirVall; 50: 71-98. 
Vieira PC, Mafezoli J, Biavatti MW. (2001). Inseticidas de Origem Vegetal. In: Ferreira, J. T. B.; Corrêa, A. G.; Vieira, P. C. (org.). Produtos Naturais no Controle de Insetos. $1^{\mathrm{a}}$ ed. São Carlos: Editora da UFSCar. Cap. 2: 23 - 45.

Vilches, A., Gil Pérez, D., Toscano, J.C. y Macías, O. (2009). “Tecnociencia para la sostenibilidad", Artículo en Internet: http://www.oei.es/decada/accion003.htm

WHO. 1990. Public Health Impact of Pesticides Used in Agriculture. World Health Organization, Geneva: 88. 